\title{
In reply: What is the appropriate definition for vulnerability to identify silent pre-frail patients?
}

\author{
Han Ting Wang, MD, MSc (D) - François Martin Carrier, MD, MSc
}

Received: 14 July 2020/Revised: 14 July 2020/Accepted: 14 July 2020/Published online: 26 August 2020

(c) Canadian Anesthesiologists' Society 2020

\section{To the Editor,}

Even as the concept of frailty is increasingly being described, it remains an elusive one to clearly define. As Drs Le Maguet and Gueret astutely pointed out, ${ }^{1}$ we described different frailty instruments that have moderate agreement at best. ${ }^{2}$ Similarly, the pre-frail or vulnerable state suffers from varying definition, making its use and interpretation challenging. Nevertheless, if we consider vulnerability as a transitional state toward frailty, vulnerable patients are "less ill" than frail patients are. This is reflected in our study by the higher proportion of American Society of Anesthesiologists physical status score $\geq 3$ among frail patients (53.3\%) compared with vulnerable patients $(42.5 \%)$, even though the median Charlson Comorbidity Index did not differ. ${ }^{3}$ Hence, vulnerability probably has less impact on clinical outcomes then frailty does. Nevertheless, with only 87 vulnerable patients included, this relationship was difficult to tease out.

The reply letter is related to letter 20-00591.

H. T. Wang, MD, MSc ( $\varangle)$

Department of Medicine, Division of Internal and Critical Care

Medicine, Hôpital Maisonneuve-Rosemont, Université de

Montréal, Montreal, QC, Canada

e-mail: ht.wang85@gmail.com

F. M. Carrier, MD, MSc

Department of Anesthesia and Department of Medicine, Critical Care Division, Centre hospitalier de l'Université de Montréal,

Montréal, Canada

Department of Anesthesiology and Pain Medicine, Université de Montréal, Centre de recherche du CHUM, Montréal, QC, Canada
Since the Clinical Frailty Scale (CFS) has a more subjective interpretation then the Fried phenotype, we thought that some patients might have underestimated their frailty state. In our sensitivity analysis, by reclassifying patients with CFS $=3$ as vulnerable, we probably introduced some more robust patients into our vulnerable category, which diluted and reduced the observed effect of vulnerability on our clinical outcomes. Although this sensitivity analysis had more power than the main analysis, its purpose was to explore the effect of potential vulnerability misclassification.

Duration of postoperative hospital length of stay (LOS) is dependent on the type of surgery performed. For every surgical specialty, minor procedures have a shorter LOS compared with major ones. Similarly, in our study, performing a major surgery had the strongest effect on LOS (adjusted odds ratio, 2.37). We did not explore how frailty and vulnerability may interact with the invasiveness of surgical procedures (i.e., effect modification). For example, vulnerability might not be important for very minor surgeries, such as hernia repairs. To our knowledge, such effect modification has not been assessed but should be further evaluated.

As mentioned by Dr. Le Maguet and Dr. Gueret, approximately one out of three patients could not be included in our study. This is the result of us being unable to reach a significant number of patients prior to their perioperative visit, even with three to four phone calls per potential participant in our recruitment process. Without any data on these patients, it is difficult to estimate their frailty states. Some patients needed surgery with a short delay (e.g., cancer surgery). These patients were added to the perioperative clinic on a short notice, which made them difficult to recruit. They might have skewed the frailty distribution since the prevalence of frailty is generally 
higher among cancer patients. Nevertheless, this remains unknown since surgeons may have fast tracked more robust patients to surgery. Nonetheless, we agree that more studies are needed to assess the impact of vulnerability on perioperative care and postoperative outcomes and to develop tools to better identify vulnerable patients that may benefit from different perioperative interventions.

Disclosures None.

Funding statement None.

Editorial responsibility This submission was handled by Dr. Hilary P. Grocott, Editor-in-Chief, Canadian Journal of Anesthesia.

\section{References}

1. Le Maguet P, Gueret $G$. What is the appropriate definition for vulnerability to identify silent pre-frail patients? Can J Anesth 2020; https://doi.org/10.1007/s12630-020-01796-0.

2. Aguayo GA, Donneau AF, Vaillant MT, et al. Agreement between 35 published frailty scores in the general population. Am J Epidemiol 2017; 186: 420-34.

3. Wang HT, Carrier FM, Tremblay A, et al. Outcomes of vulnerable elderly patients undergoing elective major surgery: a prospective cohort study. Can J Anesth 2020; 67: 847-56.

Publisher's Note Springer Nature remains neutral with regard to jurisdictional claims in published maps and institutional affiliations. 